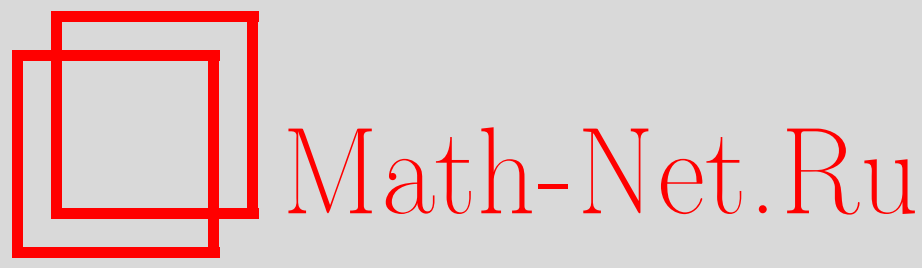

А. Р. Итс, В. Е. Корепин, Обобщенная энтропия гейзенберговской спиновой цепочки, ТМФ, 2010, том 164, номер 3, 363-367

DOI: https://doi.org/10.4213/tmf6545

Использование Общероссийского математического портала Math-Net.Ru подразумевает, что вы прочитали и согласны с пользовательским соглашением http://www.mathnet.ru/rus/agreement

Параметры загрузки:

IP : 3.80 .181 .102

26 апреля 2023 г., 14:59:44

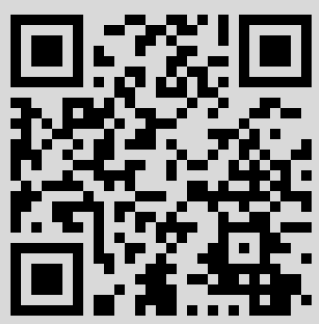




\section{ОБОБЩЕННАЯ ЭНТРОПИЯ ГЕЙЗЕНБЕРГОВСКОЙ СПИНОВОЙ ЦЕПОЧКИ}

Рассматривается квантовая $X Y$-цепочка в поперечном магнитном поле. Исследуется энтропия Реньи для блока соседних спинов в бесконечной цепочке при нулевой температуре. По сути энтропия Реньи представляет собой след матрицы плотности блока, возведенной в некоторую степень $\alpha$. Энтропия блоков большого размера вычислена в терминах эллиптической $\lambda$-функции Клейна. Предельная энтропия исследуется как функция параметра $\alpha$. Показано, что энтропия Реньи есть функция, автоморфная относительно некоторой подгруппы модулярной группы. С помощью этого наблюдения выводятся трансформационные свойства энтропии Реньи относительно отображения $\alpha \rightarrow \alpha^{-1}$.

Ключевые слова: квантовое зацепление, спиновая цепочка, анзац Бете.

\section{1. ВВЕДЕНИЕ}

В работах [1]-[5] была решена $X Y$-модель

$$
H=-\sum_{n=-\infty}^{\infty}(1+\gamma) \sigma_{n}^{x} \sigma_{n+1}^{x}+(1-\gamma) \sigma_{n}^{y} \sigma_{n+1}^{y}+h \sigma_{n}^{z} .
$$

Спектр этой модели имеет вид

$$
\epsilon_{k}=4 \sqrt{\left(\cos k-\frac{h}{2}\right)^{2}+\gamma^{2} \sin ^{2} k}
$$

Наша задача состоит в том, чтобы вычислить энтропию блока большого размера, составленного из соседних спинов и находящегося в основном состоянии $|\mathrm{GS}\rangle$, как меру зацепления между этим блоком и остатком цепочки [6]-[8].

Будем рассматривать полную цепочку как бинарную систему: $|\mathrm{GS}\rangle=|A \& B\rangle$. Блоку из $L$ соседних спинов отвечает подсистема А, а остатку цепочки - подсистема В. Матрица плотности основного состояния обозначается через $\rho_{A B}=|\mathrm{GS}\rangle\langle\mathrm{GS}|$.

${ }^{*}$ Department of Mathematical Sciences, Indiana University-Purdue University Indianapolis, Indianapolis, USA. E-mail: itsa@math.iupui.edu

${ }^{\dagger}$ C. N. Yang Institute for Theoretical Physics, State University of New York, Stony Brook, USA. E-mail: korepin@insti.physics.sunysb.edu 
Редуцированная матрица плотности подсистемы А имеет вид $\rho_{A}=\operatorname{Tr}_{B}\left(\rho_{A B}\right)$. При этом выражения для энтропии фон Неймана $S\left(\rho_{A}\right)$ и энтропии Реньи $S_{\mathrm{R}}\left(\rho_{A}, \alpha\right)$ спинового блока записываются как

$$
S\left(\rho_{A}\right)=-\operatorname{Tr}\left(\rho_{A} \ln \rho_{A}\right), \quad S_{\mathrm{R}}\left(\rho_{A}, \alpha\right)=\frac{1}{1-\alpha} \ln \operatorname{Tr}\left(\rho_{A}^{\alpha}\right), \quad 1>\alpha>0 .
$$

Здесь степень $\alpha$ выступает в роли параметра.

С помощью метода теплицевых определителей, формулы Фишера-Гартвига и методов, основанных на интегрируемых фредгольмовых операторах [9]-[14], энтропия Реньи была вычислена в работах [15]-[19]. Чтобы описать результат, необходимо ввести параметр $k$ :

$$
k= \begin{cases}\frac{\sqrt{(h / 2)^{2}+\gamma^{2}-1}}{\gamma}, & 4\left(1-\gamma^{2}\right)<h^{2}<4, \\ \sqrt{\frac{1-(h / 2)^{2}-\gamma^{2}}{1-(h / 2)^{2}},} & h^{2}<4\left(1-\gamma^{2}\right), \\ \frac{\gamma}{\sqrt{(h / 2)^{2}+\gamma^{2}-1}}, & h>2 .\end{cases}
$$

Нам также понадобятся полный эллиптический интеграл первого рода и модуль:

$$
I(k)=\int_{0}^{1} \frac{d x}{\sqrt{\left(1-x^{2}\right)\left(1-k^{2} x^{2}\right)}}, \quad \tau_{0}=\frac{I\left(k^{\prime}\right)}{I(k)},
$$

где $k^{\prime}=\sqrt{1-k^{2}}$.

Стандартный модулярный параметр $\tau$ задается соотношениями

$$
q=e^{\pi i \tau}, \quad \tau=i \frac{I\left(k^{\prime}\right)}{I(k)} \equiv i \tau_{0}, \quad \operatorname{Im} \tau>0 .
$$

Тета-функции $\theta_{j}(z, q)$ [20], [21] при этом записываются как $\theta_{j}(z \mid \tau):=\theta_{j}\left(z, e^{\pi i \tau}\right)$, $j=1,2,3,4$, а энтропия Реньи приобретает вид

$$
S_{\mathrm{R}}\left(\rho_{A}, \alpha\right)=\frac{1}{6} \cdot \frac{\alpha}{1-\alpha} \ln \left(k k^{\prime}\right)-\frac{1}{3} \cdot \frac{1}{1-\alpha} \ln \frac{\theta_{2}\left(0 \mid \alpha i \tau_{0}\right) \theta_{4}\left(0 \mid \alpha i \tau_{0}\right)}{\theta_{3}^{2}\left(0 \mid \alpha i \tau_{0}\right)}+\frac{1}{3} \ln 2 .
$$

\section{2. ЭНТРОПИЯ РЕНЬИ И МОДУЛЯРНЫЕ ФУНКЦИИ}

Квадрат эллиптического параметра $k$ как функция модулярного параметра $\tau$ стандартным образом обозначается через $\lambda(\tau)$ и называется эллиптической $\lambda$-функиией, или $\lambda$-модулярной функиией [22]-[26]. Заметим, что

$$
\lambda(\tau)=\frac{\theta_{2}^{4}(0 \mid \tau)}{\theta_{3}^{4}(0 \mid \tau)} \equiv k^{2}\left(e^{i \pi \tau}\right), \quad \operatorname{Im} \tau>0, \quad 1-\lambda(\tau)=\frac{\theta_{4}^{4}(0 \mid \tau)}{\theta_{3}^{4}(0 \mid \tau)} \equiv k^{\prime 2}\left(e^{i \pi \tau}\right) .
$$

Функция $\lambda(\tau)$ играет центральную роль в теории модулярных функций и модулярных форм, и ей посвящена обширная литература. Формулы для энтропии Реньи в терминах модулярных $\lambda$-функций имеют вид

$$
S_{\mathrm{R}}\left(\rho_{A}, \alpha\right)=\frac{1}{6} \cdot \frac{\alpha}{1-\alpha} \ln \left(k k^{\prime}\right)-\frac{1}{12} \cdot \frac{1}{1-\alpha} \ln \left\{\lambda\left(\alpha i \tau_{0}\right)\left(1-\lambda\left(\alpha i \tau_{0}\right)\right)\right\}+\frac{1}{3} \ln 2
$$


при $h>2$ и

$$
S_{\mathrm{R}}\left(\rho_{A}, \alpha\right)=\frac{1}{6} \cdot \frac{\alpha}{1-\alpha} \ln \left(\frac{k^{\prime}}{k^{2}}\right)+\frac{1}{12} \cdot \frac{1}{1-\alpha} \ln \frac{\lambda^{2}\left(\alpha i \tau_{0}\right)}{1-\lambda\left(\alpha i \tau_{0}\right)}+\frac{1}{3} \ln 2
$$

при $h<2$. Эти соотношения позволяют использовать мощный аппарат теории модулярных функций в исследованиях энтропии Реньи. Подробный анализ этого вопроса будет проведен в последующих публикациях. В настоящей работе мы ограничимся описанием двух наиболее непосредственных приложений теории модулярных функций, связанных со свойствами симметрии $\lambda$-функции.

Модулярные преобразования. Положим

$$
f(\tau):=\lambda(\tau)(1-\lambda(\tau)), \quad g(\tau):=\frac{\lambda^{2}(\tau)}{1-\lambda(\tau)}
$$

и перепишем формулы для энтропии Реньи в новом виде:

$$
S_{\mathrm{R}}\left(\rho_{A}, \alpha\right)= \begin{cases}\frac{1}{6} \cdot \frac{\alpha}{1-\alpha} \ln \left(k k^{\prime}\right)-\frac{1}{12} \cdot \frac{1}{1-\alpha} \ln f\left(\alpha i \tau_{0}\right)+\frac{1}{3} \ln 2, & h>2 \\ \frac{1}{6} \cdot \frac{\alpha}{1-\alpha} \ln \left(\frac{k^{\prime}}{k^{2}}\right)+\frac{1}{12} \cdot \frac{1}{1-\alpha} \ln g\left(\alpha i \tau_{0}\right)+\frac{1}{3} \ln 2, & h<2 .\end{cases}
$$

Из указанных симметрий вытекают соотношения симметрий для функций $f(\tau)$ и $g(\tau)$ под действием модулярной группы:

$$
\begin{array}{ll}
f(\tau+1)=-\frac{g(\tau)}{f(\tau)}, & f\left(-\frac{1}{\tau}\right)=f(\tau), \\
g(\tau+1)=g(\tau), & g\left(-\frac{1}{\tau}\right)=\frac{g(\tau)}{f(\tau)} .
\end{array}
$$

Отсюда видно, что функция $f(\tau)$ автоморфна относительно подгруппы модулярной группы, порождаемой преобразованиями

$$
\tau \rightarrow \tau+2, \quad \tau \rightarrow-\frac{1}{\tau}
$$

в то время как функция $g(\tau)$ является автоморфной относительно подгруппы модулярной группы, порождаемой преобразованиями

$$
\tau \rightarrow \tau+1, \quad \tau \rightarrow-\frac{\tau}{2 \tau+1} .
$$

Разумеется, обе функции наследуют от $\lambda$-функции свойство автоморфности относительно подгруппы, которая представляет собой общую подгруппу для подгрупп преобразований (1) и (2). Мы приходим к следующему утверждению.

ПРЕДЛОЖЕНИЕ. С точностъю до тривиалъных аддитивных членов и мультипликативных факторов и после простого масштабного преобразования энтропия Ренъи как функиия от а представляет собой функцию, автоморфную относительно подгруппы (1) группы модулярных преобразований в случае $h>2$ и автоморбную относительно подгруппы (2) группы модулярных преобразований в случае $h<2 ; 8$ обоих случаях энтропия автоморфна относительно действия общей подгрупы двух указанных групп преобразований. 
Из симметрии энтропии Реньи вытекают, в частности, следующие явные соотношения между величинами энтропии в точках $\alpha$ и $1 / \alpha \tau_{0}^{2}$ :

$$
S_{\mathrm{R}}\left(\rho_{A}, \frac{1}{\alpha \tau_{0}^{2}}\right)=\frac{\alpha \tau_{0}^{2}}{\alpha \tau_{0}^{2}-1}(1-\alpha) S_{\mathrm{R}}\left(\rho_{A}, \alpha\right)+\frac{1}{6} \cdot \frac{1-\alpha^{2} \tau_{0}^{2}}{\alpha \tau_{0}^{2}-1} \ln \frac{k k^{\prime}}{4}
$$

при $h>2$ и

$$
\begin{aligned}
S_{\mathrm{R}}\left(\rho_{A}, \frac{1}{\alpha \tau_{0}^{2}}\right)= & \frac{\alpha \tau_{0}^{2}-\alpha^{2} \tau_{0}^{2}}{\alpha \tau_{0}^{2}-1} S_{\mathrm{R}}\left(\rho_{A}, \alpha\right)+\frac{1}{6} \cdot \frac{1-\alpha^{2} \tau_{0}^{2}}{\alpha \tau_{0}^{2}-1} \ln \frac{k^{\prime}}{4 k^{2}}- \\
& -\frac{1}{12} \cdot \frac{\alpha \tau_{0}^{2}}{\alpha \tau_{0}^{2}-1} \ln f\left(\alpha i \tau_{0}\right)
\end{aligned}
$$

при $h<2$. Особо отметим появление дополнительного члена, содержащего модулярную функцию $f(\tau)$, в случае $h<2$.

\section{Список литературы}

[1] E. Lieb, T. Schultz, D. Mattis, Ann. Phys., 16 (1961), 407-466.

[2] E. Barouch, B. M. McCoy, Phys. Rev. A, 3:2 (1971), 786-804.

[3] E. Barouch, B. M. McCoy, M. Dresden, Phys. Rev. A, 2:3 (1970), 1075-1092.

[4] D. B. Abraham, E. Barouch, G. Gallavotti, A. Martin-Löf, Phys. Rev. Lett., 25:20 (1970), 1449-1450; Stud. Appl. Math., 50 (1971), 121; 51 (1972), 211.

[5] G. Müller, R.E. Shrock, Phys. Rev. B, 32:9 (1985), 5845-5850; J. Kurmann, H. Thomas, G. Müller, Phys. A, 112:1-2 (1982), 235-255.

[6] C. H. Bennett, H. J. Bernstein, S. Popescu, B. Schumacher, Phys. Rev. A, 53:4 (1996), 2046-2052, arXiv: quant-ph/9511030.

[7] A. Rényi, Probability Theory, North-Holland Ser. Appl. Math. Mech., 10, Amsterdam, North-Holland, 1970.

[8] S. Abe, A. K. Rajagopal, Phys. Rev. A, 60:5 (1999), 3461-3466, arXiv: quant-ph/9904088.

[9] B.-Q. Jin, V.E. Korepin, J. Stat. Phys., 116:1-4 (2004), 79-95, arXiv: quant-ph/0304108.

[10] A. R. Its, B.-Q. Jin, V.E. Korepin, J. Phys. A, 38:13 (2005), 2975-2990, arXiv: quant-ph/0409027.

[11] M.E. Fisher, R. E. Hartwig, "Toeplitz determinants: some applications, theorems, and conjectures", Stochastic Processes in Chemical Physics, Adv. Chem. Phys., 15, ed. K. E. Shuler, 1968, 333-353.

[12] E. L. Basor, Indiana Univ. Math. J., 28:6 (1979), 975-983.

[13] E. L. Basor, C. A. Tracy, Phys. A, 177:1-3 (1991), 167-173.

[14] A. Böttcher, B. Silbermann, Analysis of Toeplitz Operators, Springer, Berlin, 1990.

[15] A. R. Its, B.-Q. Jin, V.E. Korepin, J. Phys. A, 38:13 (2005), 2975-2990, arXiv: quant-ph/0409027.

[16] A.R. Its, B.-Q. Jin, V.E. Korepin, "Entropy of $X Y$ spin chain and block Toeplitz determinants", Universality and Renormalization, Fields Inst. Commun., 50, eds. I. Binder, D. Kreimer, AMS, Providence, RI, 2007, 151-183, arXiv: quant-ph/0606178.

[17] F. Franchini, A. R. Its, B.-Q. Jin, V.E. Korepin, Analysis of entropy of XY spin chain, arXiv: quant-ph/0606240.

[18] F. Franchini, A. R. Its, B.-Q. Jin, V.E. Korepin, J. Phys. A, 40:29 (2007), 8467-8478, arXiv: quant-ph/0609098.

[19] J. P. Keating, F. Mezzadri, Comm. Math. Phys., 252:1-3 (2004), 543-579, arXiv: quant-ph/0407047.

[20] Г. Бейтмен, А. Эрдейи, Высшие трансцендентные функции, Справочная математическая библиотека, Наука, М., 1967. 
[21] Э. Т. Уиттекер, Дж.Н. Ватсон, Курс современного анализа. Ч. 2. Трансцендентные функиии, М., 1963.

[22] F. Klein, R. Fricke, Vorlesungen über die Theorie der elliptischen Modulfunktionen. v. 2, Teubner, Leipzig, 1890.

[23] Н. И. Ахиезер, Элементы теории эллиптических функиий, Наука, М., 1970.

[24] L. Ahlfors, Complex Analysis, Internat. Ser. Pure Appl. Math., McGraw-Hill, New York, 1978.

[25] E. W. Weisstein, Elliptic lambda function. http://mathworld. wolfram.com/EllipticLambdaFunction.html

[26] E. W. Weisstein, Klein's absolute invariant. http://mathworld.wolfram.com/KleinsAbsoluteInvariant.html 